\title{
Dynamic cerebral autoregulation is an independent outcome predictor of acute ischemic stroke after endovascular therapy
}

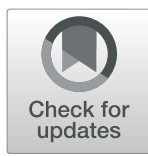

\author{
Ge Tian, Zhong Ji, Kaibin Huang, Zhenzhou Lin, Suyue Pan ${ }^{*}$ and Yongming Wu*
}

\begin{abstract}
Background: Endovascular therapy (EVT) is increasingly used to improve cerebral reperfusion after moderate-tosevere acute ischemic stroke (AIS). However, the influence of hemodynamic factors on clinical outcome is still unclear after EVT. Dynamic cerebral autoregulation (dCA) is an important brain reserve mechanism and is impaired after AIS. This study aimed to explore the role of dCA in predicting the outcome of AIS patients after EVT.

Methods: AIS patients with severe stenosis/occlusion of unilateral middle cerebral artery (MCA) or internal carotid and treatment with EVT were enrolled to receive dCA examinations at the $24 \mathrm{~h}, 72 \mathrm{~h}$ and 7 th day after stroke onset. Healthy volunteers were also recruited as controls. DCA was recorded from spontaneous fluctuations of blood pressure and MCA flow velocity. Transfer function analysis was used to derive dCA parameters, including phase difference (PD) and coherence in the low-frequency range $(0.06-0.12 \mathrm{~Hz})$. The clinical outcome was measured using the modified Rankin Scale (mRS) at 90 days after onset. Multivariate logistic regression was performed to reveal the correlation between dCA and clinical outcomes. The receiver operation characteristics (ROC) curve was performed to determine the cut-off point of PD.
\end{abstract}

Results: A total of 62 AIS patients and 77 healthy controls were included. Compared with controls, dCA were impaired bilaterally till to 7th day after onset in patients, presenting as much lower PD value on the ipsilateral side. During followup, we found that PD on the ipsilateral side at $24 \mathrm{~h}$ after onset was significantly lower in patients with unfavourable outcome $(n=41)$ than those with favourable outcome $(n=21)$, even after adjustment of confounding factors $(p=0.009)$. ROC curve analysis revealed that PD $<26.93^{\circ}$ was an independent predictor of unfavourable-outcome.

Conclusion: In AIS patients after EVT, dCA was impaired on both sides over the first 7 days. PD on the ipsilateral side at $24 \mathrm{~h}$ after onset is an independent unfavourable-outcome predictor for AIS after EVT.

Keywords: Dynamic cerebral autoregulation, Risk factors, Acute ischemic stroke, Endovascular therapy, Outcome, Predictor

\section{Background}

Acute ischemic stroke (AIS) is a leading cause of death and adult disability worldwide [1]. In Asian populations, large intracranial artery occlusive disease is the most common cause of ischemic stroke [2, 3]. Endovascular therapy (EVT) has been repeatedly proved to improve cerebral

\footnotetext{
*Correspondence: pansuyue@126.com; WU_YONGMINGNANFANG@126.com Department of Neurology, Nanfang Hospital, Southern Medical University, Guangzhou, China
}

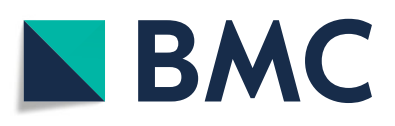

(c) The Author(s). 2020 Open Access This article is licensed under a Creative Commons Attribution 4.0 International License, which permits use, sharing, adaptation, distribution and reproduction in any medium or format, as long as you give appropriate credit to the original author(s) and the source, provide a link to the Creative Commons licence, and indicate if changes were made. The images or other third party material in this article are included in the article's Creative Commons licence, unless indicated otherwise in a credit line to the material. If material is not included in the article's Creative Commons licence and your intended use is not permitted by statutory regulation or exceeds the permitted use, you will need to obtain permission directly from the copyright holder. To view a copy of this licence, visit http://creativecommons.org/licenses/by/4.0/ The Creative Commons Public Domain Dedication waiver (http://creativecommons.org/publicdomain/zero/1.0/) applies to the data made available in this article, unless otherwise stated in a credit line to the data. arteries in [4-8]. EVT can achieve a complete revascularization ratio of $67-88 \%$ [4-8]. However, about $56 \%$ of patients remain to experience poor outcome even after endovascular treatment [9]. The causes for the lack of improvement in those cases remain incompletely understood [10].

Dynamic cerebral autoregulation $(\mathrm{dCA})$ is a pivotal mechanism to maintain stable cerebral hemodynamics [11]. 
It is regarded as an intrinsic protective mechanism of the brain, which ensures relatively constant cerebral blood flow (CBF) despite fluctuations in arterial blood pressure (ABP) or cerebral perfusion pressure [12]. However, dCA may be impaired or even vanish after ischemic stroke [13]. That means under pathological conditions that dCA was dysfunctional, $\mathrm{CBF}$ tends to passively vary with changes in $\mathrm{ABP}$, leading to brain edema, intracranial hypertension, and consequential deterioration of neurological functions and clinical outcomes [14]. Since EVT mainly changes cerebral hemodynamics in AIS, we speculated that the integrity of $\mathrm{dCA}$ might affect the prognosis of patients after EVT through regulating cerebral hemodynamics. If the correlation between $\mathrm{dCA}$ and outcome is established, dCA may be used as an early hemodynamic marker to guide early management in patients who received EVT.

In this study, we sought to assess the dysfunctional pattern of dCA in AIS patients who received EVT. Then we aimed to explore the correlation between dCA and outcome for AIS patients treated with EVT, to find out whether that $\mathrm{dCA}$ is an independent outcome predictor and to determine the cut-off point to provide a novel prognostic tool.

\section{Methods}

\section{Participants}

The study was approved by the Medical Ethics Committee of Nanfang Hospital. Written informed consent was obtained from all participants. During October 2017 to February 2019, AIS patients were recruited if they: (1) aged between 18 and 85 years; (2) had a baseline National Institutes of Health Stroke Scale (NIHSS) scores of 4 to 24; (3) had an acute, unilateral side severe stenosis/occlusion of middle cerebral artery (MCA) or internal carotid; (4) underwent EVT within $24 \mathrm{~h}$ after the onset of symptoms [15]; (5) had a Thrombolysis In Cerebral Infarction (TICI) score [16] of $\geq 2 \mathrm{~b}$ after EVT; (6) had a sufficient bilateral temporal bone window for insonation of MCA. Patients who were diagnosed as cancer or mental diseases were excluded. Patients received intravenous t-PA treatment before EVT were also regarded as eligible if the t-PA treatment was in a standard dose $(0.9 \mathrm{mg} / \mathrm{kg}$ body weight $)$ and given with $10 \%$ as a bolus and the remainder infused over $1 \mathrm{~h}$ (maximum dose, $90 \mathrm{mg}$ ). EVT decisions were based on the NIHSS score, occlusion site according to magnetic resonance angiography (MRA), and Arterial Spin Labeling (ASL) mismatch. EVT was performed by interventional neurologists using Solitaire (Solitaire, Covidien/ev3, Dublin, Ireland; FR revascularization device). The angiographic procedure had to begin and be completed within $24 \mathrm{~h}$ after the onset of stroke. If the patient had not received intravenous tPA, heparin infusion was started intravenously with a 2000-unit bolus, followed by an infusion of 450 units per hour during EVT, and was discontinued at the end of the procedure $[17,18]$. We set up a healthy volunteers' database who attended the annual physical examination in Nanfang Hospital from March 2017 to May 2017. In this study, we recruited healthy controls who were age-matched with AIS patients from the healthy volunteers' database. They were without cerebrovascular risk factors and also should meet the following inclusion criteria: (1) without intracranial and extracranial vascular stenosis by transcranial Doppler sonography (TCD), carotid artery color Doppler (CD) examination; (2) had a sufficient bilateral temporal bone window for insonation of MCAs; (3) the absence of atrial fibrillation, hyperlipidemia, hypertension, diabetes mellitus and cerebral vascular disease history; (4) without a history of chronic physical or mental diseases, without an infectious disease in the past month, without a history of smoking or heavy drinking, no being pregnant or lactating.

The clinical prognosis was assessed with the modified Rankin Scale (mRS) [19], with mRS of 2 or less defined as a favourable outcome at 90 days. All mRS assessments at 90 days after stroke onset were performed by two investigators who were unaware of the study protocol.

\section{Dynamic cerebral autoregulation (dCA) protocol}

The dCA examination protocol was performed according to the white paper from the International Cerebral Autoregulation Research Network [20]. All healthy control subjects were asked to avoid nicotine, caffeine, alcohol, and all kinds of sleep medicines for at least $24 \mathrm{~h}$ before the $\mathrm{dCA}$ examination. The examination was performed bedside with minimal surrounding stimuli. The control subjects and the patients rested in a supine position with uncrossed legs for more than $15 \mathrm{~min}$ before the examination. First, the baseline arterial blood pressure (ABP) was measured at the brachial artery using an automatic blood pressure monitor (Omron 711). Second, we simultaneously recorded continuous spontaneous ABP via a servocontrolled plethysmograph placed around the left middle finger held at the level of the heart (Finometer Pro, Netherlands) and continuous MCA blood flow velocity (BFV) at a depth of $45 \mathrm{~mm}$ to $60 \mathrm{~mm}$ with $2 \mathrm{MHz}$ probes attached to a customized head frame (EMS-9 PB, Shenzhen, China). Meanwhile, the $\mathrm{PaCO}_{2}$ level was also monitored, maintaining in stable rang. Data were recorded for $15 \mathrm{~min}$ for further data examination analysis. The artifacts were manually removed after recording.

The dCA analysis was performed using the multimodal real-time analysis software ICM+ invented by Brain Physics Lab of Cambridge University. According to the continuous ABP signal and bilateral MCA blood flow recordings, autoregulation indices including phase difference (PD) and coherence between the two signal components in the specific frequency domain range (0.06-0.12 $\mathrm{Hz}$ ) was calculated by the transfer function. We used 
coherence as a data quality control parameter, and only when the coherence was greater than 0.4 , the data were included in the subsequent statistical analysis.

\section{Statistical evaluation}

Continuous variables with normal distribution were presented as mean \pm standard deviation, and non-normally distributed continuous variables were presented as median (interquartile range, IQR). Kolmogorov-Smirnov analysis was used to test the normality of data distribution. Frequencies (percentages) were measured for categorical variables. Dynamic CA data of healthy controls were analyzed based on the mean value of the left and right cerebral sides. To assess intergroup differences, we used Student's t-test (normally distributed), Wilcoxon test (not normally distributed) and Chi-square or Fisher's exact test as appropriate. To determine the relationship between studied variables, analyses of correlation was used. A multivariate logistic regression model was constructed. We derived crude and adjusted odds ratios of the magnitude of PD and an unfavourable clinical outcome from logistic regression. Odds ratios were adjusted for confounding variables, which were different between the 2 subgroups in univariate analysis $(p<0.1)$, including Fast blood glucose, triglyceride (TG), fast blood glue, body mass index (BMI), C-reactive protein (CRP), and NIHSS on admission. $P$ values $<0.05$ were considered statistically significant. The receiver operation characteristics (ROC) curve was used to get the cutoff point of PD to predict the unfavourable outcome after EVT. All statistical calculations were performed using SPSS 19 (SPSS, Chicago, IL, USA).

\section{Results}

\section{Participant characteristics}

In 71 AIS patients, 2 cases were EVT failure, 2 cases were diagnosed as cancer, and 5 cases refused to receive EVT. A total of 62 AIS patients (55.6 \pm 14.5 years; $73 \%$ males) who underwent EVT were enrolled in the study (Table 1). Of them, 23 (37\%) received t-PA treatment before EVT. All the patients had severe stenosis/occlusion of a unilateral side of MCA $(n=41)$ or internal carotid $(n=21)$. We also recruited 77 healthy volunteers as controls (Table 1). There were no significant differences in gender, age, BMI and TG between the AIS patients and controls. However, AIS patients were associated with higher systolic blood pressure (SBP), diastolic blood pressure (DBP), fast blood glucose, heart rate and CReactive protein (CRP) compared with healthy controls (all $p<0.05$ ).

\section{Dynamic CA in controls and AIS patients}

Dynamic CA was assessed at $24 \mathrm{~h}, 72 \mathrm{~h}$, and $7 \mathrm{~d}$ after stroke onset in all AIS patients, but only once in healthy controls. Compared with healthy controls, PD values on bilateral hemispheres were significantly lower in AIS patients at different time points (Table 2). In AIS patients, $\mathrm{PD}$ values on the ipsilateral side were significantly lower than that on the contralateral side at $24 \mathrm{~h}$ and $7 \mathrm{~d}$ after onset. At $72 \mathrm{~h}$, there was a trend towards lower values of $\mathrm{PD}$ on the ipsilateral side compared with the contralateral side, although no statistical significance was reached $(p=0.066)$ (Table 2). Univariate linear regression analysis showed that fast blood glucose on admission was associated with PD on the ipsilateral hemisphere at $24 \mathrm{~h}$ after symptom onset, $\beta=-4.453, p=0.009$.

Table 1 The demographic and clinical characteristics of healthy controls and patients

\begin{tabular}{llll}
\hline Variable & Healthy controls $(\boldsymbol{n}=77)$ & Acute ischemic stroke patients $(\boldsymbol{n}=62)$ & $\boldsymbol{P}$-value \\
\hline Gender (male/female) & $53 / 24$ & $45 / 17$ & 0.710 \\
Age (years) & $55.75 \pm 11.32$ & $55.61 \pm 14.53$ & 0.949 \\
SBP (mmHg) on admission & $119.54 \pm 13.12$ & $137.85 \pm 22.15$ & $<0.001^{*}$ \\
DBP (mmHg) on admission & $72.22 \pm 10.62$ & $77.43 \pm 16.60$ & $0.031^{*}$ \\
Fast blood glucose (mmol/L) & $5.82 \pm 1.55$ & $7.29 \pm 2.40$ & $<0.001^{*}$ \\
Heart rate (bpm) & $68.09 \pm 8.71$ & $75.71 \pm 17.82$ & $0.038^{*}$ \\
BMl & $24.03 \pm 2.65$ & $23.49 \pm 3.19$ & 0.288 \\
TG (mmol/L) & $1.05 \pm 0.28$ & $1.24 \pm 0.67$ & 0.083 \\
CRP (mg/L) & $0.60(0.33,1.20)$ & $7.89(2.19,20.47)$ & $<0.001^{*}$ \\
Smoker & $0(0)$ & $8(38.10)$ & - \\
Drinker & $0(0)$ & $4(19.05)$ & - \\
Hypertension & $0(0)$ & $8(38.10)$ & - \\
Diabetes mellitus & $0(0)$ & $2(9.52)$ & - \\
\hline Vals ar & & &
\end{tabular}

Values are expressed as mean \pm SD or numbers (\%) or median (inter- quartile range, IQR)

SBP Systolic blood pressure, DBP Diastolic blood pressure, TG Triglyceride, BMI Body Mass Index, CRP Hypersensitive C-Reactive Protein, NA Not applicable

*: significant difference in comparing with control

$P$-value: $p$-value of comparing between healthy controls and patients 


\section{Dynamic CA in AIS patients with favourable and unfavourable outcomes}

During follow up, 21 (34\%) patients had favourable outcome and 41 (66\%) patients had unfavourable outcome at 90 days after stroke onset (Table 3). Unfavourable outcome patients were more likely to have higher NIHSS, fast blood glucose, BMI, TG and CRP compared with those with favourable outcome (all $p<0.05$ ). However, the ratio of $\mathrm{t}$ PA treatment and ICA stenosis/occlusion, duration from symptoms onset to hospital and the door to needle time (DNT) were all comparable between the 90-day favourable and unfavourable outcome groups (all $p>0.05$ ).

The dCA values in the favourable outcome and unfavourable outcome patients were presented in Table 4 . On the contralateral side, the PD value showed no significant difference between these two groups at different time-points (all $p>0.05$ ). On the ipsilateral side, however, the PD value at $24 \mathrm{~h}$ and $72 \mathrm{~h}$ were significantly lower in patients with unfavourable outcome than those with favourable outcome (all $p<0.05$ ). Conversely, no significant differences were observed in $7 \mathrm{~d}$ after onset.

After adjusting the confounding factors including fast blood glucose, triglyceride (TG), body mass index (BMI), C-reactive protein (CRP), and NIHSS on admission, logistic regression analysis showed that PD value on the ipsilateral hemisphere at $24 \mathrm{~h}$ after onset was an independent predictor of clinical outcome, (adjusted OR $=0.889,95 \%$ CI: 0.813-0.971, $P=0.009$ ) (Table 5, Fig. 1). Lower ipsilateral PD value at $24 \mathrm{~h}$ after onset was correlated with higher $\mathrm{mRS}(r=-0.402, P=0.007)$, indicating poorer clinical outcomes. The ROC curve was performed to determine the cutoff point that optimized the sensitivity and specificity associated with clinical outcomes. The optimal cutoff value of the ipsilateral PD for predicting unfavourable outcomes was $<26.93^{\circ}$ (sensitivity $76.50 \%$, specificity $69.20 \%$ ) (Fig. 2). The area under the curve of the ROC curve was 0.781 .

\section{Discussion}

Endovascular therapy is increasingly used to treat patients with occlusions of the large intracranial arteries. However, the effect factors on clinical outcome after EVT are still unclear. In the study, we found that dCA was impaired bilaterally over the first 7 days after symptom onset, even those patients receiving EVT. The impairment of dCA on the ipsilateral side at $24 \mathrm{~h}$ after onset was associated with clinical outcome in AIS patients who received EVT. We also determined the optimal cutoff value of the dCA index for favourabe 90-day outcome prediction.

In the study, we enrolled patients who received EVT with or without t-PA treatment previously. We found similar safety outcomes and no significant difference in functional independence with EVT after intravenous t$\mathrm{PA}$, as compared with without t-PA treatment. That may be attributed to that we determine EVT patients according to the NIHSS score, MRA, and DWI-fluid attenuated inversion recovery mismatch. Further large sample size research is needed.

\section{Choosing dCA as the marker}

Recently, numerous studies emphasize the significant predictive value of cerebrovascular reserve in ischemic stroke [21, 22]. Reduced cerebrovascular reserve indicates that the arteriolar vasodilation activity is not able to maintain stable blood flow properly in the region of cerebral hypoperfusion [21, 23, 24]. DCA is the pivot pathophysiological process to maintain stable cerebrovascular reserve capacity. It was reported that impaired dCA might trigger ischemic brain lesions [14, 25]. The underlying pathophysiological mechanisms might be that the severe stenosis may significantly reduce cerebral perfusion pressure, which could reach its lower limit for autoregulation. Under these conditions, even slight reductions of BP may result in inadequate cerebral perfusion and would not be able to protect the brain from hemodynamic ischemic events [26]. With the development of TCD monitoring, dCA can be evaluated by simultaneous assessment of spontaneous fluctuations in $\mathrm{BP}$ and BFV in MCA (assessed by TCD) using different testing modalities in the time and frequency domain [20, 27]. The methodology of such an approach based on the concept that cerebral autoregulatory system functions as a high-pass filter, which means that high-frequency fluctuations of BP are normally passing through BFV unimpededly while low-frequency oscillations are dampened. As a result, slow waves of BFV do not occur simultaneously to similar waves of BP but with a time delay. A $\mathrm{PD}$ of $0^{\circ}$ indicates total absence of autoregulation, while a

Table 2 The cerebral autoregulation value in controls and patients

\begin{tabular}{|c|c|c|c|c|}
\hline \multirow[t]{2}{*}{ Variable } & \multirow{2}{*}{$\begin{array}{l}\text { Healthy } \\
\text { controls }\end{array}$} & \multicolumn{2}{|l|}{ AIS Patients } & \multirow{2}{*}{$\begin{array}{l}\boldsymbol{P} \text { - } \\
\text { value }\end{array}$} \\
\hline & & contralateral side & ipsilateral side & \\
\hline Phase on $24 \mathrm{~h}$ after onset & $62.00 \pm 18.43$ & $40.50 \pm 28.11^{a}$ & $29.07 \pm 25.71^{a}$ & $0.012^{\sharp}$ \\
\hline Phase on $72 \mathrm{~h}$ after onset & $62.00 \pm 18.43$ & $31.71 \pm 23.88^{a}$ & $26.37 \pm 21.86^{a}$ & 0.066 \\
\hline Phase on $7 d$ after onset & $62.00 \pm 18.43$ & $37.08 \pm 27.06^{\mathrm{a}}$ & $29.13 \pm 22.37^{a}$ & $0.020^{\sharp}$ \\
\hline
\end{tabular}

asignificant difference in comparing between controls and patients

\#significant difference in comparing the dynamic cerebral autoregulation value between the contralateral side and the ipsilateral side

$P$ : $P$-value of comparing between the contralateral side and the ipsilateral side 
Table 3 The demographic and clinical characteristics of patients with 90-day favourable outcome (mRS of 0-2) and unfavourable outcome (mRS of 3-6)

\begin{tabular}{|c|c|c|c|}
\hline Variable & Favourable outcome $(\boldsymbol{n}=21)$ & Unfavourable outcome $(\boldsymbol{n}=41)$ & $\boldsymbol{P}$-value \\
\hline Gender (male/female) & $17 / 4$ & $28 / 13$ & 0.375 \\
\hline Age (years) & $51.48 \pm 11.23$ & $57.73 \pm 15.67$ & 0.109 \\
\hline SBP $(\mathrm{mmHg})$ on admission & $134.10 \pm 20.69$ & $139.73 \pm 22.87$ & 0.358 \\
\hline DBP (mmHg) on admission & $78.05 \pm 16.28$ & $77.13 \pm 15.45$ & 0.831 \\
\hline Fast blood glucose (mmol/L) & $6.06 \pm 1.88$ & $7.92 \pm 2.41$ & $0.003^{*}$ \\
\hline Heart rate (bpm) & $74.38 \pm 14.50$ & $75.71 \pm 17.82$ & 0.792 \\
\hline BMI & $22.18 \pm 3.14$ & $24.16 \pm 3.03$ & $0.019^{*}$ \\
\hline TG (mmol/L) & $0.98 \pm 0.30$ & $1.37 \pm 0.75$ & $0.018^{*}$ \\
\hline CRP (mg/L) & $3.70(1.15,7.13)$ & $10.96(4.57,26.00)$ & $0.003^{*}$ \\
\hline Smoker & $8(38.10)$ & $19(46.34)$ & 0.596 \\
\hline Drinker & $4(19.05)$ & $13(31.71)$ & 0.375 \\
\hline Hypertension & $8(38.10)$ & $18(43.90)$ & 0.788 \\
\hline Diabetes mellitus & $2(9.52)$ & $11(26.83)$ & 0.187 \\
\hline NIHSS on admission & $9(5,14)$ & $15(11,20)$ & $<0.001^{*}$ \\
\hline Large artery stenosis/occlusion & & & 0.777 \\
\hline MCA & $13(61.90)$ & $28(68.29)$ & \\
\hline ICA & $8(38.10)$ & $13(31.71)$ & \\
\hline t-PA treatment & $9(42.86)$ & $14(34.15)$ & 0.583 \\
\hline Onset to hospital time (min) & $348.88 \pm 280.57$ & $288.33 \pm 180.33$ & 0.372 \\
\hline DNT (min) & $115.06 \pm 49.05$ & $95.53 \pm 58.58$ & 0.258 \\
\hline The time intervals between completed EVT and dCA measurement (hour) & $9.33 \pm 3.57$ & $7.76 \pm 3.75$ & 0.117 \\
\hline
\end{tabular}

Values are expressed as mean \pm SD or numbers (\%) or median (inter- quartile range, IQR)

SBP Systolic blood pressure, DBP Diastolic blood pressure, TG Triglyceride, BMI Body Mass Index, CRP Hypersensitive C-Reactive Protein, NIHSS National Institutes of Health Stroke Scale, MCA Middle cerebral artery, ICA Internal carotid artery, DNT Door to needle time, $d C A$ dynamic cerebral autoregulation, EVT Endovascular therapy

${ }^{*}$ significant difference in comparing between favourable-outcome group and unfavourable-outcome group

$P$-value: $p$-value of comparing between favourable-outcome group and unfavourable-outcome group

large positive PD of $40^{\circ}-70^{\circ} \mathrm{can}$ be regarded as intact autoregulation. In our study, the PD obtained from oscillations in $\mathrm{ABP}$ and $\mathrm{CBFV}$ for MCA in healthy subjects were similar, which is consistent with previous studies. Comparing with healthy controls, we found that dCA on both the ipsilateral hemisphere and the contralateral hemisphere were impaired in AIS patients. Consistently with our study, previous researches also reported bilateral impaired CA in the acute stroke [28, 29]. The mechanism of this trans-hemispheric communication may be

Table 4 The dynamic cerebral autoregulation value in favourable outcome and unfavourable outcome patients

\begin{tabular}{|c|c|c|c|}
\hline Variable & Favourable outcome $(\boldsymbol{n}=21)$ & Unfavourable outcome $(\boldsymbol{n}=41)$ & $\boldsymbol{P}$-value \\
\hline \multicolumn{4}{|l|}{ Phase on $24 \mathrm{~h}$ after onset } \\
\hline Phase on contralateral side & $51.97 \pm 33.58$ & $34.92 \pm 23.59^{a}$ & 0.072 \\
\hline Phase on ipsilateral side & $45.47 \pm 27.67^{\mathrm{a}}$ & $20.64 \pm 20.32^{a}$ & $0.001^{\#}$ \\
\hline \multicolumn{4}{|l|}{ Phase on $72 \mathrm{~h}$ after onset } \\
\hline Phase on contralateral side & $39.97 \pm 28.35^{a}$ & $26.20 \pm 18.93^{a}$ & 0.066 \\
\hline Phase on ipsilateral side & $34.70 \pm 21.06^{\mathrm{a}}$ & $21.09 \pm 21.01^{\mathrm{a}}$ & $0.032^{\#}$ \\
\hline \multicolumn{4}{|l|}{ Phase on $7 d$ after onset } \\
\hline Phase on contralateral side & $45.59 \pm 28.65^{\mathrm{a}}$ & $31.23 \pm 25.14^{\mathrm{a}}$ & 0.180 \\
\hline Phase on ipsilateral side & $39.74 \pm 24.57^{\mathrm{a}}$ & $22.49 \pm 18.71^{\mathrm{a}}$ & 0.054 \\
\hline
\end{tabular}

${ }^{a}$ significant difference in comparing with control after adjusting confounding factors

\#: significant difference in comparing between favourable-outcome group and unfavourable-outcome group

$P$ : $P$-value of comparing between favourable-outcome group and unfavourable-outcome group 
Table 5 Multivariate analysis of clinical characteristics and dynamic cerebral autoregulation for favorable long-term outcome

\begin{tabular}{|c|c|c|c|c|}
\hline Variables & Crude OR (95\% Cl) & $\boldsymbol{P}$-value & Adjusted OR (95\% Cl) & $\boldsymbol{P}$-value \\
\hline \multicolumn{5}{|l|}{ Clinical factors } \\
\hline Fast blood glucose $(\mathrm{mmol} / \mathrm{L})$ & $1.726(1.171,2.544)$ & $0.006^{*}$ & $3.523(1.255,9.885)$ & $0.017^{*}$ \\
\hline BMI & $1.250(1.027,1.521)$ & $0.026^{*}$ & & 0.623 \\
\hline $\mathrm{TG}(\mathrm{mmol} / \mathrm{L})$ & $3.401(0.937,12.343)$ & 0.063 & & 0.760 \\
\hline CRP $(\mathrm{mg} / \mathrm{L})$ & $1.088(1.008,0.175)$ & $0.031^{*}$ & & 0.162 \\
\hline NIHSS on admission & $1.276(1.105,1.474)$ & $0.001^{*}$ & $1.825(1.155,2.886)$ & $0.010^{*}$ \\
\hline \multicolumn{5}{|l|}{ Cerebral autoregulation } \\
\hline \multicolumn{5}{|l|}{$\mathrm{dCA}$ on $24 \mathrm{~h}$ after onset } \\
\hline Phase on ipsilateral side & $0.955(0.926,0.985)$ & $0.004^{*}$ & $0.889(0.813,0.971)$ & $0.009^{*}$ \\
\hline Phase on contralateral side & $0.978(0.956,1.000)$ & $0.048^{*}$ & & 0.184 \\
\hline \multicolumn{5}{|l|}{$\mathrm{dCA}$ on $72 \mathrm{~h}$ after onset } \\
\hline Phase on ipsilateral side & $0.970(0.942,0.998)$ & $0.039^{*}$ & & 0.205 \\
\hline Phase on contralateral side & $0.974(0.948,1.000)$ & 0.053 & & 0.183 \\
\hline \multicolumn{5}{|l|}{ dCA on 7d after onset } \\
\hline Phase on ipsilateral side & $0.962(0.923,1.003)$ & 0.069 & & 0.259 \\
\hline Phase on contralateral side & $0.979(0.950,1.010)$ & 0.180 & & \\
\hline
\end{tabular}

TG Triglyceride, BMI Body Mass Index, CRP Hypersensitive C-Reactive Protein, NIHSS National Institutes of Health Stroke Scale

"significant difference in comparing between favourable-outcome group and unfavourable-outcome group, $p<0.05$

diaschisis where there is distant functional depression due to the effects of loss of axons (mainly facilitatory) arising at the site of the lesion and, in the case of the cerebral hemispheres, these may synapse with neurons in the contralateral hemisphere via the corpus callosum [30]. However, further basic research was needed to confirm it. In addition, Dawson et al., have indicated that $\mathrm{dCA}$ appeared impaired bilaterally and remained so for at least 1 to 2 weeks over the subacute post-stroke period [31]. In a follow-up study, CA was also abnormal on the affected side $>2$ months after stroke onset [32]. All those were closely in keeping with our finding, that

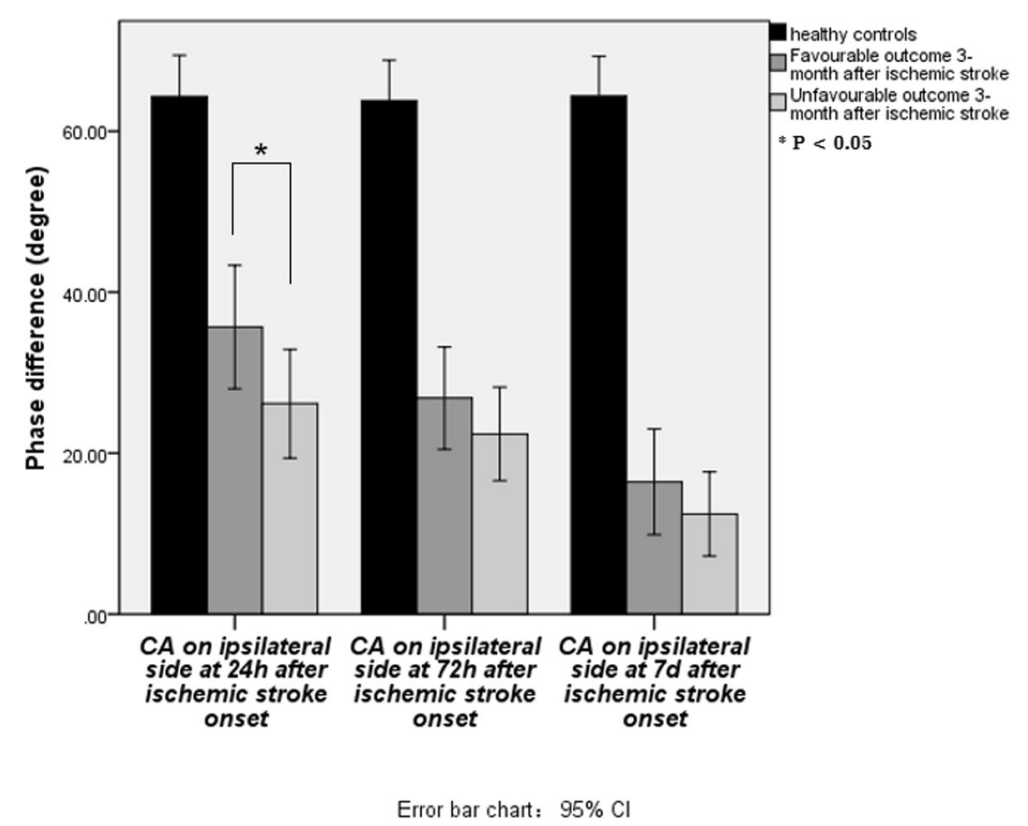

Fig. 1 On the ipsilateral side, however, the PD value at $24 \mathrm{~h}$ was significantly lower in patients with unfavourable outcome than those with favourable outcome 


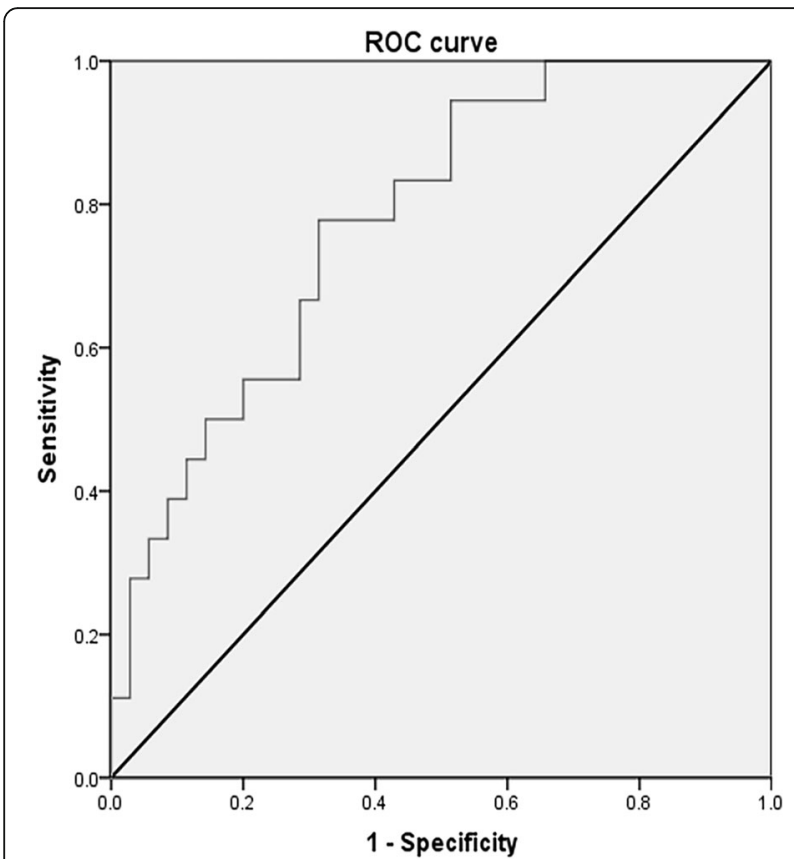

Fig. 2 The ROC curve the optimal cutoff value of the ipsilateral PD for predicting unfavourable outcomes was $<26.93^{\circ}$ (sensitivity $76.50 \%$, specificity $69.20 \%$ )

the dysautoregulation still existed 7 days after symptom onset, even receiving EVT. That means our results support the theory of an additional secondary autoregulatory failure in acute cerebral ischemia. However, due to time restriction of EVT, we could not assess preoperative $\mathrm{dCA}$, we still do not understand the detail change of postoperative dCA through comparing with preoperative dCA.

\section{Influence factors on clinical outcome after EVT}

In our study, we observed significantly lower dCA on the ipsilateral side at $24 \mathrm{~h}$ after symptom onset in unfavourable-outcome patients than favourable-outcome patients even after adjusting confounding factors. The $t$ test showed contralateral dCA with a difference bordering on significance between favourable-outcome and unfavourable-outcome groups. However after adjusting confounding factors, no significance of contralateral $\mathrm{dCA}$ was found. Larger sample size research is needed to explore it in future. The ROC showed that the PD value on the ipsilateral side at $24 \mathrm{~h}$ lower than $26.93^{\circ}$ indicated unfavourable 90-day-outcome in patients who received EVT. In the studies about AIS patients performed by Chi et al. [33] and Castro el al [34], PD values were associated with mRS score $>2$ at 3 months in patients with moderate to severe stroke even in the multivariate analysis. Castro et al. suggested $\mathrm{PD}<37^{\circ}$ as a cutoff to predict unfavourable outcomes, whereas Chi et al. suggested a cutoff of $\mathrm{PD}<61^{\circ}$. The difference may be because of the different frequency band selection in transfer function analysis (TFA), that Chi et al. analyze $\mathrm{PD}$ in very low frequency. Besides, we noticed that our cut-off PD value was lower than the value reported by previous research. That may indicate that EVT may improve the tolerance to hemodynamic fluctuations. Saur et al. [35] and Reinhard et al. [25] suggested that the development of cerebral dysautoregulation may be particularly critical during the first days of reperfusion in larger infarctions. The first day is probably also the most critical period with regard to functional brain reorganization. It might be explained that in the acute stage after EVT, hemodynamic status changed. Focal dysautoregulation in the ischemic core and the former penumbral area might enhance the reperfusion injury and lead to secondary endothelial dysfunction, losing the protective function for the ischemic penumbra. Our findings indicate that special attention should be paid to the early acute stage of large acute ischemic stroke when secondary autoregulatory failure can evolve mainly in the affected vascular territory. In patients treated with EVT, BP control strategies in large ischemic stroke should be guided by the status of vessel recanalization and autoregulatory capacity, especially at $24 \mathrm{~h}$ after ischemic stroke onset. Consistent with our finding although with a different dCA monitoring measurement, a recent research suggested that continuous estimation of autoregulation-based treatment strategies after EVT was feasible and could provide a BP range for individual patients tailored to their own physiology [22]. In future, larger sample size research is needed to observe dCA status when changing BP level.

The present analysis has some limitations. Firstly, we recruited more male subjects than female in our study, since there are more male stroke patients than female in China [36], and female subjects are more likely to have insufficient bilateral temporal bone windows for insonation due to the low density of the temporal bone [37]. Secondly, the sample size of our research is small, further larger sample size research is needed. Thirdly, in our study, we used heparin during EVT, which might be the limitation of the protocol. Further relative research is needed.

\section{Conclusion}

In conclusion, dCA are impaired on the ipsilateral hemisphere and contralateral hemisphere during the first 7 days after AIS symptom onset. Dynamic CA on the ipsilateral hemisphere at $24 \mathrm{~h}$ after symptom onset is independently associated with clinical outcomes in AIS patients who received EVT. Impaired dCA can be set up as an early hemodynamic marker to guide acute stage management in patients who received EVT. It provides a novel prognostic tool to improve clinical outcomes achieving personalized treatment. 


\section{Abbreviations}

EVT: Endovascular therapy; AIS: Acute ischemic stroke; dCA: Dynamic cerebral autoregulation; MCA: Middle cerebral artery; PD: Phase difference; mRS: Modified Rankin Scale; ROC: Receiver operation characteristics; CBF: Cerebral blood flow; ABP: Arterial blood pressure; NIHSS: National Institutes of Health Stroke Scale; TICl: Thrombolysis In Cerebral Infarction; MRA: Magnetic resonance angiography; ASL: Arterial Spin Labeling; TCD: Transcranial doppler sonography; CD: Carotid artery color doppler; IQR: Interquartile range; TG: Triglyceride; BMI: Body mass index; CRP: Creactive protein; SBP: Systolic blood pressure; DBP: Diastolic blood pressure; TFA: Transfer function analysis

\section{Acknowledgments}

The work was funded by the National Natural Science Foundation of China (Grant No. 81801179).

\section{Authors' contributions}

Study concept and design: WYM, PSY, TG. Acquisition of data: TG, JZ, LZZ Statistical analysis: HKB, LZZ. Drafting of the manuscript: TG, HKB. Critical revision of the manuscript for important intellectual content: TG, WYM, JZ, HKB. Study supervision: WYM, PSY. The authors read and approved the final manuscript.

\section{Funding}

The work was funded by the National Natural Science Foundation of China (Grant No. 81801179).

\section{Availability of data and materials}

The raw/processed data required to reproduce these findings cannot be shared at this time as the data also forms part of an ongoing study.

\section{Ethics approval and consent to participate}

The study was approved by the Medical Ethics Committee of Nanfang Hospital. Written informed consent was obtained from all participants. The data presented were collected for the purpose of quality assurance and, thus, the identity of the individual patients were anonymous.

\section{Consent for publication}

Not applicable.

\section{Competing interests}

The authors declare no competing financial interests.

Received: 7 February 2020 Accepted: 20 April 2020

\section{Published online: 15 May 2020}

\section{References}

1. Bonita R, Mendis S, Truelsen T, Bogousslavsky J, Toole J, Yatsu F. The global stroke initiative. Lancet Neurol. 2004;3:391-3.

2. Wong LK. Global burden of intracranial atherosclerosis. Int J Stroke. 2006;1: 158-9.

3. Gorelick PB, Wong KS, Bae HJ, Pandey DK. Large artery intracranial occlusive disease: a large worldwide burden but a relatively neglected frontier. Stroke. 2008:39:2396-9.

4. Goyal M, Demchuk AM, Menon BK, Eesa M, Rempel JL, Thornton J, et al. Randomized assessment of rapid endovascular treatment of ischemic stroke. N Engl J Med. 2015;372:1019-30.

5. Campbell BC, Mitchell PJ, Kleinig TJ, Dewey HM, Churilov L, Yassi N, et al. Endovascular therapy for ischemic stroke with perfusion-imaging selection. N Engl J Med. 2015;372:1009-18.

6. Berkhemer OA, Fransen PS, Beumer D, van den Berg LA, Lingsma HF, Yoo $\mathrm{A}$, et al. A randomized trial of intraarterial treatment for acute ischemic stroke. N Engl J Med. 2015;372:11-20.

7. Jovin TG, Chamorro A, Cobo E, de Miquel MA, Molina CA, Rovira A, et al. Thrombectomy within 8 hours after symptom onset in ischemic stroke. $N$ Engl J Med. 2015;372:2296-306.

8. Saver JL, Goyal M, Bonafe A, Diener HC, Levy El, Pereira VM, et al. Stentretriever thrombectomy after intravenous t-PA vs. T-PA alone in stroke. N Engl J Med. 2015;372:2285-95.
9. Rodrigues FB, Neves JB, Caldeira D, Ferro JM, Ferreira JJ, Costa J. Endovascular treatment versus medical care alone for ischaemic stroke: systematic review and meta-analysis. BMJ. 2016;353:i1754.

10. Rabinstein AA, Albers GW, Brinjikji W, Koch S. Factors that may contribute to poor outcome despite good reperfusion after acute endovascular stroke therapy. Int J Stroke. 2018;14(1):23.

11. Xiong L, Liu X, Shang T, Smielewski P, Donnelly J, Guo ZN, et al. Impaired cerebral autoregulation: measurement and application to stroke. J Neurol Neurosurg Psychiatry. 2017;88:520-31.

12. van Beek AH, Claassen JA, Rikkert MG, Jansen RW. Cerebral autoregulation: an overview of current concepts and methodology with special focus on the elderly. J Cereb Blood Flow Metab. 2008;28:1071-85.

13. Immink RV, van Montfrans GA, Stam J, Karemaker JM, Diamant M, van Lieshout JJ. Dynamic cerebral autoregulation in acute lacunar and middle cerebral artery territory ischemic stroke. Stroke. 2005:36:2595-600.

14. Reinhard M, Rutsch S, Lambeck J, Wihler C, Czosnyka M, Weiller C, et al. Dynamic cerebral autoregulation associates with infarct size and outcome after ischemic stroke. Acta Neurol Scand. 2012;125:156-62.

15. Powers WJ, Rabinstein AA, Ackerson T, Adeoye OM, Bambakidis NC, Becker K, et al. 2018 guidelines for the early management of patients with acute ischemic stroke: a guideline for healthcare professionals from the american heart association/American stroke association. Stroke. 2018;49:e46-e110.

16. Tomsick T. TIMI, TIBI, TICl: I came, I saw, I got confused. AJNR Am J Neuroradiol. 2007;28:382-4

17. Broderick JP, Palesch YY, Demchuk AM, Yeatts SD, Khatri P, Hill MD, et al, Endovascular therapy after intravenous t-PA versus t-PA alone for stroke. N Engl J Med. 2013;368:893-903.

18. Khatri P, Yeatts SD, Mazighi M, Broderick JP, Liebeskind DS, Demchuk AM, et al. Time to angiographic reperfusion and clinical outcome after acute ischaemic stroke: an analysis of data from the interventional Management of Stroke (IMS III) phase 3 trial. Lancet Neurol. 2014;13:567-74.

19. van Swieten JC, Koudstaal PJ, Visser MC, Schouten HJ, van Gijn J. Interobserver agreement for the assessment of handicap in stroke patients. Stroke. 1988;19:604-7.

20. Claassen JA, Meel-van DAA, Simpson DM, Panerai RB. Transfer function analysis of dynamic cerebral autoregulation: a white paper from the international cerebral autoregulation research network. J Cereb Blood Flow Metab. 2016:36:665-80.

21. Marshall RS, Rundek T, Sproule DM, Fitzsimmons BF, Schwartz S, Lazar RM. Monitoring of cerebral vasodilatory capacity with transcranial Doppler carbon dioxide inhalation in patients with severe carotid artery disease. Stroke. 2003;34:945-9.

22. Petersen NH, Silverman A, Strander SM, Kodali S, Wang A, Sansing LH, et al. Fixed compared with autoregulation-oriented blood pressure thresholds after mechanical thrombectomy for ischemic stroke. Stroke. 2020:51:914-21.

23. King A, Serena J, Bornstein NM, Markus HS. Does impaired cerebrovascular reactivity predict stroke risk in asymptomatic carotid stenosis? A prospective substudy of the asymptomatic carotid emboli study. Stroke. 2011;42:1550-5.

24. Gupta A, Chazen JL, Hartman M, Delgado D, Anumula N, Shao H, et al. Cerebrovascular reserve and stroke risk in patients with carotid stenosis or occlusion: a systematic review and meta-analysis. Stroke. 2012:43:2884-91.

25. Reinhard M, Wihler C, Roth M, Harloff A, Niesen WD, Timmer J, et al. Cerebral autoregulation dynamics in acute ischemic stroke after rtPA thrombolysis. Cerebrovasc Dis. 2008;26:147-55.

26. Semenyutin VB, Asaturyan GA, Nikiforova AA, Aliev VA, Panuntsev GK, Iblyaminov VB, et al. Predictive value of dynamic cerebral autoregulation assessment in surgical management of patients with high-grade carotid artery stenosis. Front Physiol. 2017;8:872.

27. Zhang $\mathrm{R}$, Zuckerman $\mathrm{JH}$, Giller CA, Levine BD. Transfer function analysis of dynamic cerebral autoregulation in humans. Am J Phys. 1998;274:H233-41.

28. Armstead WM. Cerebral blood flow autoregulation and dysautoregulation. Anesthesiol Clin. 2016;34:465-77.

29. Xiong L, Tian G, Lin W, Wang W, Wang L, Leung T, et al. Is dynamic cerebral autoregulation bilaterally impaired after unilateral acute ischemic stroke? J Stroke Cerebrovasc Dis. 2017;26:1081-7.

30. Eames PJ, Blake MJ, Dawson SL, Panerai RB, Potter JF. Dynamic cerebral autoregulation and beat to beat blood pressure control are impaired in acute ischaemic stroke. J Neurol Neurosurg Psychiatry. 2002;72:467-72.

31. Dawson SL, Panerai RB, Potter JF. Serial changes in static and dynamic cerebral autoregulation after acute ischaemic stroke. Cerebrovasc Dis. 2003: 16:69-75. 
32. Dawson SL, Manktelow BN, Robinson TG, Panerai RB, Potter JF. Which parameters of beat-to-beat blood pressure and variability best predict early outcome after acute ischemic stroke? Stroke. 2000;31:463-8.

33. Chi NF, Hu HH, Wang CY, Chan L, Peng CK, Novak V, et al. Dynamic cerebral autoregulation is an independent functional outcome predictor of mild acute ischemic stroke. Stroke. 2018;49:2605-11.

34. Castro P, Serrador JM, Rocha I, Sorond F, Azevedo E. Efficacy of cerebral autoregulation in early ischemic stroke predicts smaller infarcts and better outcome. Front Neurol. 2017;8:113.

35. Saur D, Lange R, Baumgaertner A, Schraknepper V, Willmes K, Rijntjes M, et al. Dynamics of language reorganization after stroke. Brain. 2006;129: 1371-84.

36. Liu M, Wu B, Wang WZ, Lee LM, Zhang SH, Kong LZ. Stroke in China: epidemiology, prevention, and management strategies. Lancet Neurol. 2007; 6:456-64.

37. Wijnhoud AD, Franckena M, van der Lugt A, Koudstaal PJ, Dippel ED. Inadequate acoustical temporal bone window in patients with a transient ischemic attack or minor stroke: role of skull thickness and bone density. Ultrasound Med Biol. 2008;34:923-9.

\section{Publisher's Note}

Springer Nature remains neutral with regard to jurisdictional claims in published maps and institutional affiliations.

Ready to submit your research? Choose BMC and benefit from:

- fast, convenient online submission

- thorough peer review by experienced researchers in your field

- rapid publication on acceptance

- support for research data, including large and complex data types

- gold Open Access which fosters wider collaboration and increased citations

- maximum visibility for your research: over $100 \mathrm{M}$ website views per year

At BMC, research is always in progress.

Learn more biomedcentral.com/submissions 